\title{
Prevalence and clinical course of typical hemolytic uremic syndrome among siblings
}

\author{
Alfredo Eymann, M.D. ${ }^{a}$, Paula Coccia, M.D. ${ }^{b}$, Claudia Raddavero, M.D. ${ }^{b}$, Gabriela Lafi, M.D. ${ }^{b}$, \\ Verónica Ferraris, M.D. ${ }^{b}$, José Ramírez, M.D. ${ }^{b+}$ and Jorge Ferraris, M.D. ${ }^{b}$
}

\begin{abstract}
Introduction. Hemolyticuremicsyndrome(HUS) is aninfectious disease caused by Shiga toxin-producing Escherichia coli. The objective of this study was to assess the risk of transmission and clinical course between siblings with typical HUS.

Population and methods. Medical records of children with typical HUS between 1997 and 2012 were reviewed. Sibling pairs were established as inclusion criteria. A severity score was defined.

Results. A total of 133 patients with HUS were recorded; 40 had siblings and 4 progressed to HUS (10\%). The mean age of the 4 sibling pairs was 29.3 months old $(\mathrm{SD} \pm 11.5) ; 5(62.5 \%)$ were girls. The mean time between each case was 5.7 days (SD \pm 3 ). HUS was more severe in the siblings who became infected in the second place.

Conclusion. The risk of HUS transmission between siblings was $10 \%$, and the clinical course of the second sibling was less favorable.

Key words: hemolytic uremic syndrome, siblings, Escherichia coli, risk, Argentina.
\end{abstract}

http:/ /dx.doi.org/10.5546/aap.2016.eng.553

\section{INTRODUCTION}

Hemolytic uremic syndrome (HUS) is clinically defined by the clinical triad of thrombocytopenia, microangiopathic hemolytic anemia, and acute kidney failure, and is characterized by the presence of thrombotic microangiopathy in the pathological examination. In 1964, Carlos Gianantonio, M.D., published a series of cases in Argentine children and provided a full description of the clinical aspects and course of HUS. ${ }^{1}$

a. Department of Clinical Pediatrics of Hospital Italiano de Buenos Aires.

b. Department of Pediatric Nephrology of Hospital Italiano de Buenos Aires.

E-mailaddress:AlfredoEymann,M.D.:alfredo.eymann@gmail.com

Funding: None.

Conflict of interest: None.

Received: 4-14-2016

Accepted: 8-4-2016
Survival of HUS patients improved with intermittent peritoneal dialysis in the acute phase and with kidney transplantation in the chronic stage. However, HUS is still a major health problem in Argentina. It is the leading cause of acute kidney failure and the second cause of chronic kidney disease, which accounts for approximately $20 \%$ of kidney transplants in children. $^{2}$

HUS is caused, in $90 \%$ of cases, by Shiga toxinproducing Escherichia coli (STEC); this means it is an infectious disease, and is called typical HUS. Its incidence rate is variable, but in Argentina, the annual HUS incidence ranges between 10 and 12 cases every 100000 children younger than 5 years old; and it is the highest rate reported worldwide. ${ }^{3}$

Risk factors associated with the development of typical HUS include eating meat outside the house, eating undercooked meat, living in or visiting a place with farm animals, and contact with children younger than 5 years old with diarrhea. ${ }^{4}$

Cattle are the primary reservoir for STEC, and food or water contaminated with cattle feces is often the most common source of infections in Argentina.

Secondary infection through person-toperson transmission may also occur. ${ }^{5}$ It has been described that family members who come in contact with children with typical HUS commonly have STEC colonization, and Shiga toxin has been frequently identified in the members of the same family. ${ }^{6,7}$ The development and severity of person-to-person transmission may also depend on other factors, such as the amount of inoculum ingested and individual susceptibility.

Our objective was to assess the risk of transmission and clinical course between siblings with typical HUS.

\section{POPULATION AND METHODS}

The medical records of children with typical HUS admitted to the Department of Pediatrics of Hospital Italiano between March 1 ${ }^{\text {st }}, 1997$ and December 31 $1^{\text {st }}, 2012$ were reviewed. Their 
families were contacted by telephone to check recorded data.

Typical HUS was defined as the triad of thrombocytopenia, microangiopathic hemolytic anemia, and acute kidney failure after a bloody or non-bloody diarrhea episode.

As of 2000, all cases are reported to the Ministry of Health.

All patients had a stool culture to look for STEC and/or detect verotoxin-1 and -2 in feces using specific cytotoxicity of Vero cells, an enzyme immunoassay, or an immunochromatographic rapid test, depending on the year of patient admission.

Sibling pairs (6 months to 6 years old) who had typical HUS in the same epidemiological period (2-14 days) were established as inclusion criteria.

Kidney involvement was defined as an increase in serum creatinine above the normal range adjusted for age or the presence of hematuria and proteinuria in urinary sediment; thrombocytopenia was defined as a platelet count below $150 \times 10^{9} / \mathrm{L}$; and neurological involvement was established as lethargy, irritability, ataxia, seizures, or coma.

The following outcome measures were assessed: age, sex, date of onset of clinical and lab HUS signs between the first and the second siblings, lab tests at admission (white blood cell count, platelet count, hematocrit, creatinine, urinary sediment), clinical characteristics (oligoanuria, intermittent peritoneal dialysis, neurological involvement, and clinical course).

A severity score was established based on the mortality and chronic kidney involvement predictors described by Oakes et al. ${ }^{8,9}$ in 2006 and 2008:

- White blood cell count equal to 20000 cells per $\mathrm{mm}^{3}$ or higher (1) and lower (0).

- Hematocrit equal to $23 \%$ or lower (0) and higher (1).

- Oligoanuria equal to 5 days or longer (2) or shorter (1), and no oligoanuria (0).

- Presence (1) and absence (0) of neurological involvement.

A score of 0 was considered less severe, whereas 5 accounted for a more severe case.

Categorical outcome measures were analyzed by frequency and continuous outcome measures with normal distribution were studied using Student's t test. A $p$ value below 0.05 was considered significant.

\section{RESULTS}

A total of 133 patients with typical HUS were recorded. Their mean age was 24 months old (SD \pm 9.4$) ; 58 \%$ were girls. The mortality rate was

TABLE 1. Clinical characteristics, lab tests at the time of admission and severity score in the 4 pairs of siblings with hemolytic uremic syndrome

\begin{tabular}{|c|c|c|c|c|c|c|c|c|}
\hline$\underline{\text { Family }}$ & \multicolumn{2}{|c|}{1} & \multicolumn{2}{|c|}{2} & \multicolumn{2}{|c|}{3} & \multicolumn{2}{|c|}{4} \\
\hline Year of diagnosis & \multicolumn{2}{|c|}{1997} & \multicolumn{2}{|c|}{1999} & \multicolumn{2}{|c|}{2002} & \multicolumn{2}{|c|}{2006} \\
\hline Age (months) & 25 & 10 & 23 & 43 & 31 & 47 & 28 & 28 \\
\hline Sex & $\mathrm{F}$ & M & $\mathrm{M}$ & $\mathrm{F}$ & $\mathrm{M}$ & $\mathrm{F}$ & $\mathrm{F}$ & $\mathrm{F}$ \\
\hline Diagnosis & Inicial & Subs & Inicial & Subs & Inicial & Subs & Inicial & Subs \\
\hline $\begin{array}{l}\text { Time until HUS development } \\
\text { (days) }\end{array}$ & \multicolumn{2}{|c|}{9} & \multicolumn{2}{|c|}{5} & \multicolumn{2}{|c|}{2} & \multicolumn{2}{|c|}{7} \\
\hline Oligoanuria (days) & 1 & 5 & 0 & 1 & 6 & 30 & 2 & 9 \\
\hline IPD (days) & 0 & 6 & 0 & 0 & 5 & 32 & 0 & 9 \\
\hline Baseline creatinine (mg/dL) & 2.1 & 3.5 & 0.8 & 1.2 & 3.4 & 1.2 & 1.7 & 3.7 \\
\hline Hematocrit (\%) & 21 & 25 & 30 & 29.6 & 23.6 & 31 & 21 & 26 \\
\hline Platelet count (cells / mm³) & 50000 & 17200 & 130000 & 29000 & 43700 & 53800 & 64300 & 74500 \\
\hline $\begin{array}{l}\text { White blood cell count } \\
\left(\text { cells } / \mathrm{mm}^{3}\right)\end{array}$ & 12300 & 13500 & 12000 & 14600 & 13800 & 36100 & 19500 & 24500 \\
\hline Neurological involvement & Yes & Yes & No & No & No & Yes & No & Yes \\
\hline Course & Normal & Normal & Normal & Normal & Normal & $\mathrm{P}$ and $\mathrm{CKF}$ & Normal & $\mathrm{P}$ \\
\hline Severity score & 2 & 4 & 1 & 2 & 3 & 5 & 1 & 5 \\
\hline
\end{tabular}

HUS: hemolytic uremic syndrome. M: male; F: female. Normal: normal creatinine, normal blood pressure and no proteinuria. P: proteinuria. CKF: chronic kidney failure. IPD: intermittent peritoneal dialysis. Subs: subsequent. 
$1.8 \%$, and neurological involvement was the cause of death in all fatal cases.

Forty patients had siblings; 16 had diarrhea and 4 progressed to HUS (10\%). There was a pair of twin girls.

The mean age of the 4 sibling pairs was 29.3 months old $(\mathrm{SD} \pm 11.5) ; 5(62.5 \%)$ were girls. No patient had HUS recurrence, and no case occurred in adults. The mean time between HUS transmission from the first to the second sibling was 5.7 days $(S D \pm 3)$, and the mean follow-up time was 11 years $(\mathrm{SD} \pm 5.4)$.

The pairs of siblings diagnosed between 2002 and 2006 had STEC, which was confirmed by identification of verotoxin in feces.

The baseline clinical and lab characteristics of sibling pairs are described in Table 1.

Siblings who developed typical HUS in second place had a more severe score, as per the poor prognosis parameters assessed at disease initiation, with a higher frequency of neurological involvement and prolonged kidney failure. The mean severity score of siblings who developed typical HUS first was $1.75(\mathrm{SD} \pm 0.95)$ and that of siblings who had the disease in second place was $4(\mathrm{SD} \pm 1.4)(p<0.03)$.

\section{DISCUSSION}

Ten percent of siblings of primary patients developed typical HUS, and the clinical course of the second sibling was less favorable.

Family members who are in contact with children with HUS are usually colonized by STEC and seroconversion frequently occurs in the family members of these children.5,6

Although gastrointestinal symptoms in family contacts were less common in our study, it has been reported that approximately $40 \%$ of household contacts of children with HUS have the free toxin identified in their feces. ${ }^{6,7}$

HUS outbreaks may start due to simultaneous exposure of several individuals to a common food source, although secondary person-toperson transmission may occur within small communities or families. ${ }^{6,7}$

In our study, patients who became sick in the second place had a more severe clinical course. This may be associated with virulence factors, the mode of transmission or a greater amount of inoculum.

Children with typical HUS and central nervous system, gastrointestinal or myocardial involvement have a higher morbidity and mortality rate during the acute phase of HUS. ${ }^{8-10}$

Between $20 \%$ and $30 \%$ of patients have long- term kidney sequelae, including proteinuria, high blood pressure, and a reduced glomerular filtration rate. . $^{210-12}$

Several studies have attempted to establish predictors of a poor prognosis in terms of mortality and kidney disease progression in patients with typical HUS. ${ }^{8,9}$

The studies conducted by Oakes et al. in 2006 and 2008 demonstrated that leukocytosis and mild anemia at the time of admission to the hospital were associated with mortality, and the duration of oliguria and/or anuria were predictors of kidney morbidity in the long term. ${ }^{8,9}$

In endemic regions, many family cases of HUS are caused by the Shiga toxin. Cases occurring in the second sibling appeared within 4 weeks in $3.4 \%$ of studied families in Utah, USA. ${ }^{13}$

Prior studies reported that strains of STEC O157 caused sporadic typical HUS cases in Argentina, and that different members of the same family became infected with symptomatic or asymptomatic STEC. Signs of infection were observed in $31.6 \%$ of members of studied families, and parents had a higher infection rate than siblings. ${ }^{6}$

Given STEC's incubation period (median: 8 days), it is very difficult to establish a difference between co-primary cases and secondary transmission across family members with a history of common exposure.

A retrospective cohort study assessed a STEC O157 outbreak in South Wales and the United Kingdom in the fall of 2005 and observed that the presence of a sibling and a difference in age of less than 5 years with the primary case were independent predictors for families with secondary cases. It was also demonstrated that hospitalization of STEC cases reduces the risk of household transmission. ${ }^{14}$

Family cases of HUS have been reported to be associated with genetic mutations or acquired deficiencies in complement regulation. This type is called atypical HUS; it is usually recurrent and, in general, is not related to exposure to the Shiga toxin, although some families with observed mutations in the complement system had a concomitant STEC infection which had triggered HUS. ${ }^{15}$

Our study poses several limitations, including its retrospective design and the small number of sibling pairs included. However, our results show the prevalence of typical HUS among siblings in the studied period and population. The course of the second case may be more severe. 
We believe that, in the case of typical HUS diagnosis, it is necessary to provide close epidemiological surveillance of the siblings of children with HUS,

\section{REFERENCES}

1. Gianantonio CA, Vitacco M, Mendilaharzu J, Rutty A, et al. The Hemolytic-Uremic Syndrome. J Pediatr 1964;64: 478-91.

2. Spizzirri FD, Rahman RC, Bibiloni N, Ruscasso JD, et al. Childhood hemolytic uremic syndrome in Argentina: long term follow up and prognostic features. Pediatr Nephrol 1997;11(2):156-60.

3. Agencia Gubernamental de Control. ¿Qué es el síndrome urémico hemolítico o suh? [Accessed on: August $4^{\text {th }}, 2016$ ]. Available at: http://www.agcontrol.gob.ar/pdf/AGC_ InformeSUH.pdf.

4. Rivas M, Sosa-Estani S, Rangel J, Caletti MG, et al. Risk Factors for Sporadic Shiga Toxin-producing Escherichia coli Infections in Children, Argentina. Emerg Infect Dis 2008;14(5):763-71.

5. Boudailliez B, Berquin P, Mariani-Kurkdjian P, Ilef D, et al. Possible person-to-person transmission of Escherichia coli O111-associated hemolytic uremic syndrome. Pediatr Nephrol 11997;11(1):36-9.

6. Lopez EL, Diaz M, Devoto S, Grinstein S,et al. Evidence of infection with organisms producing Shiga-like toxins in household contacts of children with the hemolytic uremic syndrome. Pediatr Infect Dis J 1991;10(1):20-4.
7. Rivas M, Voyer L, Tous M, De Mena M, etal. Verocytotoxin producing Escherichia coli infection in family members of children with hemolytic uremic syndrome. Medicina ( $B$ Aires) 1996;56(2):119-25.

8. Oakes RS, Siegler RL, McReynolds MA, Pysher T, et al. Predictors of fatality in postdiarrheal hemolytic uremic syndrome. Pediatrics 2006;117(5):1656-62.

9. Oakes RS, Kirkham JK, Nelson RD, Siegler RL. Duration of oliguria and anuria as predictors of chronic renal-related sequelae in post-diarrheal hemolytic uremic syndrome. Pediatr Nephrol 2008;23(8):1303-8.

10. Rahman RC, Cobeñas CJ, Drut R, Amoreo OR, et al. Hemorrhagic colitis in postdiarrheal hemolytic uremic syndrome: retrospective analysis of 54 children. Pediatr Nephrol 2012;27(2):229-33.

11. Gianviti A, Tozzi AE, De Petris L, Caprioli A, et al. Risk factors for poor renal prognosis in children with hemolytic uremic syndrome. Pediatr Nephrol 2003;18(12):1229-35

12. Fitzpatrick MM, Shah V, Trompeter RS, Dillon MJ, et al. Long term renal outcome of childhood hemolytic uremic syndrome. BMJ 1991;303(6801):489-92.

13. Siegler RL, SherbotieJR, Denkers ND, Pavia AT.Clustering of post-diarrheal (shiga toxin mediated) hemolytic uremic syndrome in families. Clin Nephrol 2003;60(2):74-9.

14. Werber D, Mason BW, Evans MR, Salmon RL. Preventing household transmission of Shiga toxin-producing Escherichia coliO157infection: promptly separating siblings might be the key. Clin Infect Dis 2008;46(8):1189-96.

15. Campistol JM, Arias M, Ariceta G, Blasco M, etal. An update for atypical haemolytic uraemic syndrome: diagnosis and treatment. A consensus document. Nefrologia 2015;35(5): 421-47. 